\title{
Proposal for a Standard Problem for Micromagnetic Simulations Including Spin-Transfer Torque
}

\author{
Massoud Najafi, ${ }^{1,2, *}$ Benjamin Krüger, ${ }^{3, \dagger}$ Stellan Bohlens, ${ }^{3}$ Matteo Franchin, ${ }^{4}$ \\ Hans Fangohr, ${ }^{4}$ Antoine Vanhaverbeke, ${ }^{5}$ Rolf Allenspach, ${ }^{5}$ Markus Bolte, ${ }^{1,2}$ \\ Ulrich Merkt, ${ }^{2}$ Daniela Pfannkuche, ${ }^{3}$ Dietmar P. F. Möller, ${ }^{1}$ and Guido Meier ${ }^{2}$ \\ ${ }^{1}$ Arbeitsbereich Technische Informatiksysteme, Fachbereich Informatik, \\ Universität Hamburg, Vogt-Kölln-Str. 30, 22527 Hamburg, Germany \\ ${ }^{2}$ Institut für Angewandte Physik und Zentrum für Mikrostrukturforschung, \\ Universität Hamburg, Jungiusstr. 11, 20355 Hamburg, Germany \\ ${ }^{3}$ I. Institut für Theoretische Physik, Universität Hamburg, Jungiusstr. 9, 20355 Hamburg, Germany \\ ${ }^{4}$ School of Engineering Sciences, University of Southampton, SO17 1BJ, Southampton, United Kingdom \\ ${ }^{5}$ IBM Zürich Research Laboratory, Säumerstrasse 4, CH-8803 Rüschlikon, Switzerland
}

(Dated: March 27, 2009)

\begin{abstract}
The spin-transfer torque between itinerant electrons and the magnetization in a ferromagnet is of fundamental interest for the applied physics community. To investigate the spin-transfer torque, powerful simulation tools are mandatory. We propose a micromagnetic standard problem including the spin-transfer torque that can be used for the validation and falsification of micromagnetic simulation tools. The work is based on the micromagnetic model extended by the spin-transfer torque in continuously varying magnetizations as proposed by Zhang and Li. The standard problem geometry is a permalloy cuboid of $100 \mathrm{~nm}$ edge length and $10 \mathrm{~nm}$ thickness, which contains a Landau pattern with a vortex in the center of the structure. A spin-polarized dc current density of $10^{12} \mathrm{~A} / \mathrm{m}^{2}$ flows laterally through the cuboid and moves the vortex core to a new steady-state position. We show that the new vortex-core position is a sensitive measure for the correctness of micromagnetic simulators that include the spin-transfer torque. The suitability of the proposed problem as a standard problem is tested by numerical results from four different finite-difference and finite-element-based simulation tools.
\end{abstract}

PACS numbers: $75.40 . \mathrm{Mg}, 75.00 .00,85.75 .-\mathrm{d}, 72.25 . \mathrm{Ba}$

Keywords: micromagnetic simulation, spin-transfer torque, standard problem, verification of simulation tools, OOMMF, NMag, $\mathrm{M}^{3} \mathrm{~S}$

\section{INTRODUCTION}

Ferromagnets can be found in most devices that require nonvolatile storage of information. Ferromagnets have been successfully used in hard disks for more than 50 years $^{1}$. Recently the field of research has been extended to the development of nanometer-sized ferromagnetic nonvolatile storage devices that offer a high storage density accompanied by a high data rate $^{2}$. The magnetic random access memory (MRAM) has been developed as the first nanostructured ferromagnetic memory module ${ }^{3}$. An MRAM cell consists of a multilayer system with of two ferromagnetic layers separated by a non-magnetic layer. Information is stored in the orientation of the magnetization in the two ferromagnetic layers. Depending on the properties of the non-magnetic layer, the information can be read with the help of the tunnel magnetoresistance (TMR) effect ${ }^{4}$ or the giant magnetoresistance (GMR) effect ${ }^{5}$. For this, a current is applied to the multilayer. The resistance depends on the relative alignment of the magnetizations of the ferromagnetic layers. To write information in such a memory cell, a current is applied across two perpendicular wires. At the intersection of the two wires, the resulting Oersted field is strong enough to switch the magnetic orientation of the first magnetic layer, the so-called free layer. The magnetic ori- entation of the second ferromagnetic layer, the so-called pinned layer, should not change during this process ${ }^{3,6}$. The application of an Oersted field corresponds to the write process in a hard disk. As explained by Chappert et al. ${ }^{7}$, there are different restrictions using an Oersted field that limit the storage density of the MRAM. To increase the storage density, it is therefore necessary to find an alternative way to switch the magnetization.

Slonczewski ${ }^{8,9}$ and Berger ${ }^{10}$ predicted in 1996 that a spin-polarized current flowing through a ferromagnetic conductor can apply a relevant torque to its magnetization, owing to the exchange coupling between the spins of the itinerant electrons and those of the localized electrons. Since its discovery the so-called spin-transfer torque (STT) has been considered as a key to increase the storage density and lead to a new generation of storage devices, such as the spin-transfer torque random access memory (STTRAM) ${ }^{11}$ and the racetrack memory ${ }^{12}$. The STTRAM is an MRAM which uses the spin-transfer torque instead of the Oersted field for the switching process. The racetrack memory stores bits along a single ferromagnetic wire. To write and read information, a current is applied along the wire that moves the bits to a writing or reading unit.

Two theoretical descriptions of the spin-transfer torque exist: The first description has been developed by Slonczewski ${ }^{8,9}$ and describes a current traversing an in- 
terface between a ferromagnet and a non-magnetic metal and its concomitant torque on the magnetization. It can successfully describe a STTRAM. The second description has been developed by Berger ${ }^{10}$ and was later refined by Zhang and $\mathrm{Li}^{13}$ as well as by Thiaville et al. ${ }^{14}$. It deals with the spin-transfer torque in the case of a continuously varying magnetization. In this case the spin-transfer torque acts on inhomogeneous magnetization patterns, such as domain walls or magnetic vortices. Thus, also the magnetic processes in a racetrack memory ${ }^{12}$ and gyrating magnetic vortices driven by spin-transfer torque ${ }^{15,16}$ can be described.

Other memory devices such as the dynamic random access memory (DRAM) ${ }^{17}$ or the static random access memory (SRAM) ${ }^{18}$ have shown that it is necessary to develop analytical descriptions and powerful simulation tools like SPICE $^{19}$ to optimize their properties $^{2}$. The theoretical descriptions of the spintransfer torque $\mathrm{e}^{8-10,13,14}$ are the basis for devices that exploit the interaction between spin-polarized currents and magnetization. There exists a variety of simulation tools, such as the Micromagnetic Modeling and Simulation kit $\mathrm{M}^{3} \mathrm{~S}^{20}$, $\mathrm{Nmag}^{21}$, the object-oriented micromagnetic framework OOMMF ${ }^{22}, \mathrm{LLG}^{23}$, and Micromagus ${ }^{24}$, that implement the micromagnetic model ${ }^{25}$ and include the spin-transfer torque model. To compare different simulation tools the Micromagnetic Modeling Activity Group $(\mu \mathrm{Mag})^{26}$ publishes standard problems for micromagnetism. These micromagnetic problems allow the results of a simulation tool to be verified. So far, there is no standard problem that includes the spin-transfer torque. Here we propose a problem that allows the validation of micromagnetic simulation tools that implement the spintransfer torque of Berger ${ }^{10}$ with the extension by Zhang and $\mathrm{Li}^{13}$. We further present numerical solutions to the proposed problem and analytical solutions of the problem given by Krüger et al. ${ }^{27}$.

\section{PROBLEM SELECTION}

In this section, selection criteria for the standard problem are defined and possible adaptations of each criterion are given. The focus of our standard problem is the spintransfer torque extension. Thus we chose criteria that ensure the traceability of errors in the implementation of this extension. A prerequisite is that the simulation tool derives correct results for the numerical time-integration, the demagnetization field, the exchange field, and the Zeeman field.

\section{A. Selection Criteria}

To select a standard problem that is appropriate to trace errors in the spin-transfer torque extension, we first define four general selection criteria. According to the strategy of $\mu \mathrm{Mag}^{26}$, these criteria are:
1. The problem has to be specified in such a way that different simulation tools are able to reproduce the initial magnetization configuration independent of their implementation.

2. The problem has to ensure, that the reaction of the magnetization depends significantly on the current and leads to an unambiguous time evolution of the magnetization.

3. The problem has to be solvable in reasonable computation time. This is important to run the standard problem repeatedly, which is necessary to fix program errors.

4. The problem has to offer an unambiguous and characteristic measure for the magnetization dynamics and thus enables verification or falsification of a simulation tool. This measure has to be computable conveniently and independently of the implementation of the tool.

\section{B. Theoretical Background}

We use the micromagnetic model including the spintransfer torque of Berger ${ }^{10}$ with the extension by Zhang and $\mathrm{Li}^{13}$. The equation of motion of the magnetization is given by

$$
\begin{aligned}
\frac{\partial \vec{M}}{d t}= & -\gamma \vec{M} \times \vec{H}_{\mathrm{eff}}+\frac{\alpha}{M_{s}} \vec{M} \times \frac{d \vec{M}}{d t} \\
& -\frac{b_{j}}{M_{s}^{2}} \vec{M} \times(\vec{M} \times(\vec{j} \cdot \vec{\nabla}) \vec{M}) \\
& -\xi \frac{b_{j}}{M_{s}} \vec{M} \times(\vec{j} \cdot \vec{\nabla}) \vec{M}
\end{aligned}
$$

with the gyromagnetic ratio $\gamma$, the Gilbert damping parameter $\alpha$, and the saturation magnetization $M_{s}$. The effective magnetic field $\vec{H}_{\text {eff }}$ includes the external as well as the internal fields. The coupling constant between the current and the magnetization is $b_{j}=\left(P \mu_{B}\right) /\left(e M_{s}(1+\right.$ $\left.\xi^{2}\right)$ ), where $P$ denotes the spin polarization of the current density $\vec{j}, \mu_{B}$ the Bohr magneton, and $\xi=\tau_{\text {ex }} / \tau_{\text {sf }}$ the degree of non-adiabacity, which is the ratio between the exchange relaxation time $\tau_{\text {ex }}$ and the spin-flip relaxation time $\tau_{\mathrm{sf}}$. Equation (1) can be written in the explicit form

$$
\begin{aligned}
\frac{d \vec{M}}{d t}= & -\gamma^{\prime} \vec{M} \times \vec{H}_{\mathrm{eff}}-\frac{\alpha \gamma^{\prime}}{M_{s}} \vec{M} \times\left(\vec{M} \times \vec{H}_{\mathrm{eff}}\right) \\
& -\frac{b_{j}^{\prime}}{M_{s}^{2}}(1+\alpha \xi) \vec{M} \times(\vec{M} \times(\vec{j} \cdot \vec{\nabla}) \vec{M}) \\
& -\frac{b_{j}^{\prime}}{M_{s}}(\xi-\alpha) \vec{M} \times(\vec{j} \cdot \vec{\nabla}) \vec{M}
\end{aligned}
$$


with the abbreviations $\gamma^{\prime}=\gamma /\left(1+\alpha^{2}\right)$ and $b_{j}^{\prime}=b_{j} /(1+$ $\left.\alpha^{2}\right)$ as written by Krüger et al. ${ }^{28}$.

\section{Adaptation of the Criteria}

On the basis of the physical model, we define the standard problem that complies with the criteria defined above.

Criterion (i) is fulfilled by splitting the problem into two sub-problems that are computed separately. Each sub-problem is the computation of a separate simulation run. The first simulation is performed based on Eq. (2) in the absence of current $\vec{j}$. It starts from a magnetization pattern that has to be given by an equation. The resulting equilibrium magnetization is used as the initial magnetization for the second simulation with an applied current.

Criterion (ii) can be fulfilled by the selection of an inhomogeneous magnetization pattern, e.g., a domain wall or a vortex, and the selection of a spatially and temporally homogeneous current. We decided to take a permalloy cuboid with a vortex pointing upwards for the initial equilibrium state of the second sub-problem. The choice of a vortex and a spatially and temporally homogeneous current leads to an unambiguously distinguishable adiabatic and non-adiabatic reaction of the magnetization $27,29,30$. The equation of motion leads to a new steady state that provides a simple validation measure independent of the prior time evolution. In contrast, the choice of a resonant excitation of the vortex with alternating current is not suitable, because a small error in the simulated resonance frequency would drastically change the phase and amplitude of the result, which would complicate the falsification. A dc current reduces the complexity of the problem and enables the correctness of the results by the final steady state of the vortex core to be checked as a characteristic measure.

Criterion (iii) can be met by a small number of discretization points and a magnetization pattern that exhibits significant changes within few time-integration steps. The number of discretization points is given by the size of the cuboid and the average distance between the discretization points. We use a small cuboid that still can relax to a vortex state. The discretization of the permalloy cuboid must be chosen such that the vortex core is resolved. The necessary resolution is achieved if the distance between the discretization points is significantly below the exchange length $l_{\mathrm{ex}}=\sqrt{2 A /\left(\mu_{0} M_{s}^{2}\right)}$ where $A$ is the constant of the exchange interaction. To decrease the number of time-integration steps, we choose a large Gilbert damping parameter $\alpha$, so that the magnetization rapidly reaches equilibrium.

Criterion (iv) can be fulfilled by the calculation of the spatially averaged magnetization, which is proportional to the vortex-core position as shown in appendix A. Thus the motion of the vortex core is an unambiguous and characteristic measure of the magnetization dynamics $^{27}$.

\section{PROBLEM DEFINITION}

The problem is defined with the standard material parameters of permalloy ${ }^{31}$, with the exception of the Gilbert damping parameter $\alpha$. These parameters are given by an exchange constant $A=13 \cdot 10^{-12} \mathrm{~J} / \mathrm{m}$, a saturation magnetization $M_{\mathrm{s}}=8 \times 10^{5} \mathrm{~A} / \mathrm{m}$, which corresponds to an exchange length $l_{\mathrm{ex}}=5.7 \mathrm{~nm}$, and a gyromagnetic ratio $\gamma=2.211 \times 10^{5} \mathrm{~m} / \mathrm{C}$. According to criterion (iii) we select a cuboid geometry with a sample size of $100 \times 100 \times 10 \mathrm{~nm}^{3}$ in the $x$-, $y$-, and $z$ - direction, respectively. This allows the problem to be simulated with a spatial and temporal discretization, which can be computed in a few hours on an standard personal computer ${ }^{46}$. In contrast to a circular film element, the cuboid geometry simplifies the comparison of simulation tools using finite-difference (FDM) and finiteelement methods (FEM), because there are no irregular edges that are a possible source of errors in the FDM.

\section{A. Computation of the Starting Condition without Spin-Transfer Torque}

In accordance with criterion (i), the first sub-problem of the standard problem starts with an initial magnetization pattern as illustrated in Fig. 1(a). The initial vortex state relaxes into equilibrium as illustrated in Fig. 1(b). The initial magnetization pattern is chosen as

$$
\vec{M}=M_{\mathrm{s}} \cdot \frac{\vec{f}}{|\vec{f}|}, \vec{f}=\left(\begin{array}{c}
-\left(y-y_{0}\right) \\
x-x_{0} \\
R
\end{array}\right),
$$

where $\vec{r}=(x, y, z)$ is the position of the cell and $\vec{r}_{0}=\left(x_{0}, y_{0}, z_{0}\right)=(50 \mathrm{~nm}, 50 \mathrm{~nm}, 5 \mathrm{~nm})$ is the center of the cuboid. $R$ is related to the radius of the vortex and is set to $R=10 \mathrm{~nm}$ as this value leads to a short relaxation time. A Gilbert damping constant of $\alpha=1$ is chosen to obtain a fast relaxation and thus save computation time, but the relaxed equilibrium state is independent of $\alpha$. The effective field is given by the exchange and the demagnetization field. The simulation stops when the magnetization has reached an equilibrium state. The stopping criterion is $\max _{\vec{r} \in \mathcal{V}}\left|1 / M_{s} \cdot d \vec{M} / d t\right| \leq 0.01 \mathrm{rad} / \mathrm{ns}$, where $\mathcal{V}$ is the volume of the cuboid. As shown in Fig. 1(b), the equilibrium state is a vortex as required by criterion (ii). The vortex core points in the $z$-direction (positive polarization) and the in-plane magnetization curls counterclockwise (positive chirality). 




(a)

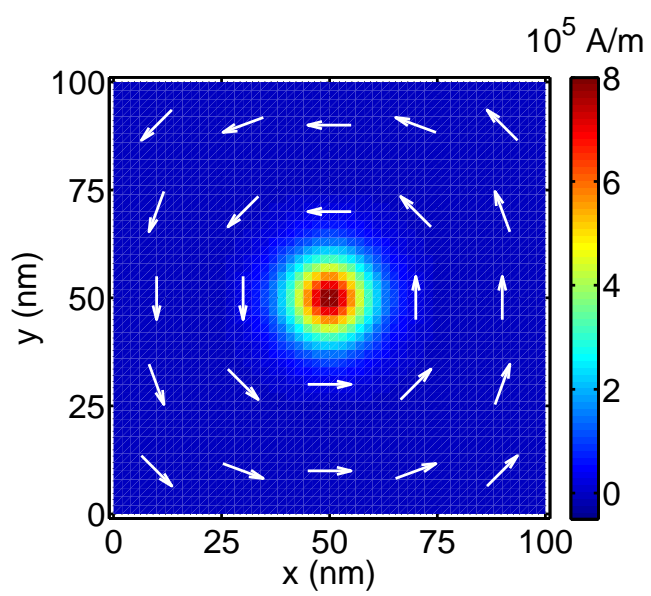

(b)

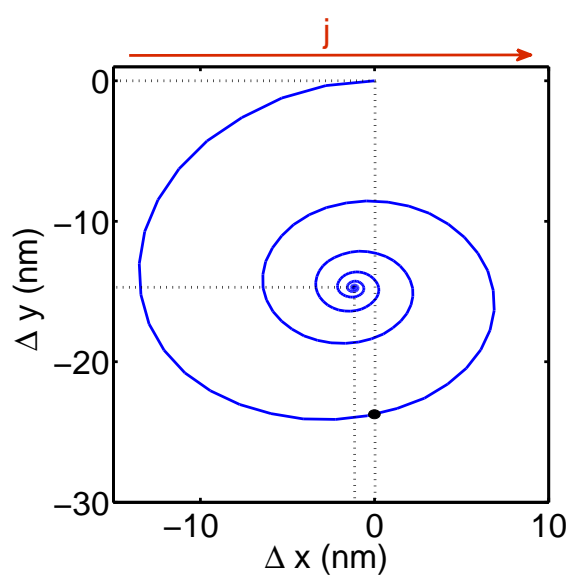

(a)

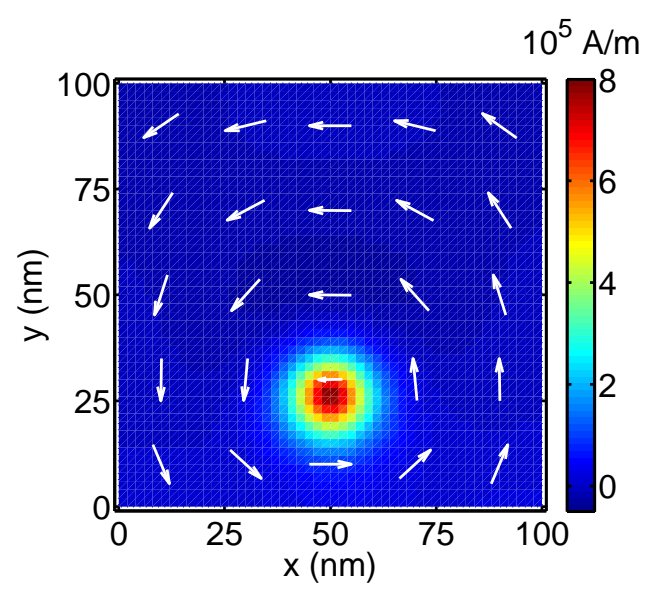

(b)
FIG. 1: (a) Initial state of the magnetization for the first subproblem as given by Eq. (3). The magnetization is averaged along the $z$-direction. The color scale shows the $z$-component of the magnetization. (b) Relaxed vortex state as initial state for the second part of the computation including the spintransfer torque. Simulations are computed with $\mathrm{M}^{3} \mathrm{~S}$.

\section{B. Computation including Spin-Transfer Torque}

The second sub-problem, which includes the spintransfer torque, starts with the equilibrium state of the first sub-problem. The effective field is the same as in the first sub-problem. As required in criterion (ii) and illustrated in Fig. 2(a), a spatially homogeneous spinpolarized dc current of $10^{12} \mathrm{~A} / \mathrm{m}^{2}$ is instantaneously applied in the $x$-direction $(\vec{j}=(j, 0,0))$, i.e., the electrons flow from right to left. The damping constant $\alpha=0.1$ of this sub-problem is chosen to obtain a reasonable fast relaxation on the one hand and enough oscillations to assist the comparison of results from different simulation packages on the other hand. The value also allows the detection of errors of the spin-transfer torque term that depend on the damping parameter
FIG. 2: (a) Two-dimensional representation of the position of the vortex core as a function of time. The dot indicates the vortex-core position at the time $t=0.73 \mathrm{~ns}$. (b) Snapshot of the magnetization of the permalloy cuboid at $t=0.73 \mathrm{~ns}$ when the vortex-core position crosses the line $\Delta x=0$ for the first time. The magnetization is excited by a homogeneous spin-polarized current density of $10^{12} \mathrm{~A} / \mathrm{m}^{2}$ in the $x$-direction, i.e., the electrons flow from right to left. The magnetization is averaged along the $z$-direction. The color scale is the same as in Fig. 1. Simulations are computed with $\mathrm{M}^{3} \mathrm{~S}$.

$\alpha$. The degree of non-adiabaticity $\xi=0.05$ is chosen to get a significant contribution of the non-adiabatic spin-transfer torque term to the final vortex-core position and to achieve a non-zero contribution of the fourth term in Eq. (2). The simulation stops when the stopping criterion $\max _{\vec{r} \in \mathcal{V}}\left|1 / M_{s} \cdot d \vec{M} / d t\right| \leq 0.01 \mathrm{rad} / \mathrm{ns}$ has been reached. To compare different simulation packages, one has to calculate the spatially averaged magnetization over time. The resulting trajectory of the simulation shows a damped rotation of the vortex core around a new steady-state position of $\Delta x=x-x_{0}=-1.2 \mathrm{~nm}$ and $\Delta y=y-y_{0}=-14.7 \mathrm{~nm}$, as illustrated in Fig. 2. 
The vortex-core position $\Delta x, \Delta y$ is related to the center of the cuboid. It is determined by averaging the magnetization along the $z$-direction and interpolating the outof-plane magnetization in the $x$ - and $y$-direction with a polynomial of second order. The position of the vortex core is then given by the maximum of this polynomial.

\section{Falsification Properties}

Suitable falsification properties as demanded in criterion (iv) are important for the development of a simulation tool. The influence of errors in the spin-transfer torque extension or an improper, i.e., too coarse, spatial discretization has been investigated for the proposed standard problem and is outlined in the following.

\section{Sensitivity to Errors in the Spin-Transfer Torque Extension}

First we analyze the influence of errors in the spintransfer torque extension. To show the sensitivity of the problem to those errors, we investigate changes of the spin-transfer torque given by a constant factor. This is emulated by a variation of the degree of non-adiabaticity $\xi$ and the current density $j$. The analytical model explained in appendix B predicts that a change of $\xi$ will linearly affect the $y$-component of the spatially averaged magnetization $\left\langle M_{y}\right\rangle$, whereas a change of $j$ will affect the $x$ - and $y$-component of the spatially averaged magnetization $\left\langle M_{x}\right\rangle$ and $\left\langle M_{y}\right\rangle$ equally. Figure 3 shows three sets of parameters for $\xi$ and $j$ that illustrate the clearly distinguishable reactions of the magnetization to a change in the adiabatic, the non-adiabatic, and the entire spintransfer torque. As a first set we chose an increased spin-transfer torque realized by an increased current density. It leads to a proportionally increased $x$ - and $y$ component $\left\langle M_{x}\right\rangle$ and $\left\langle M_{y}\right\rangle$ of the spatially averaged magnetization during its time evolution. The second set is an increased non-adiabatic spin-transfer torque created by an increased degree of non-adiabaticity $\xi$. This configuration leads to a proportionally increased $y$-component $\left\langle M_{y}\right\rangle$ of the averaged magnetization during the time evolution of the magnetization. The third set describes an decreased influence of the adiabatic spin-transfer torque term obtained by simultaneously decreasing $j$ and increasing $\xi$. This configuration induces a proportionally decreased $x$-component $\left\langle M_{x}\right\rangle$ of the spatially averaged magnetization during the time evolution of the magnetization. The results illustrate that a variation of $\xi$ and $j$ results in a clear change of the magnetization which, according to appendix B, should be linear with the change in $\xi$ and $j$. As illustrated in Fig. 3, a variation of the adiabatic spin-transfer torque by a constant factor linearly affects the $x$-component of the spatially averaged magnetization $\left\langle M_{x}\right\rangle$, whereas a variation of the non-adiabatic spin-transfer torque by a constant factor linearly affects



(a)

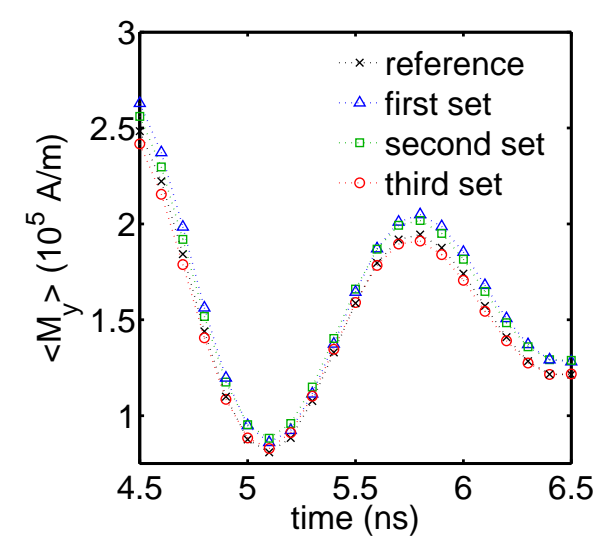

(b)

FIG. 3: (a) Spatially averaged magnetization $\left\langle M_{x}\right\rangle$ and (b) $\left\langle M_{y}\right\rangle$ for different values of $\xi$ and $j$. The crosses show the time evolution of the spatially averaged magnetization for the reference parameters $\xi=0.05$ and $j=10^{12} \mathrm{~A} / \mathrm{m}^{2}$. The triangles show the result for the first set of parameters, when the spintransfer torque parameter $j$ is increased by $5 \%$. The squares show the result of the second set, when the non-adiabatic spin-transfer torque parameter $\xi$ is increased by $5 \%$. The circles show the results of the third set, when the adiabatic spin-transfer torque is changed by a simultaneous decrease of the current density and increase of $\xi$ by $5 \%$ each. The maximum difference of the spatially averaged magnetization amounts to $14.40 \mathrm{kA} / \mathrm{m}(5.11 \%)^{a}$ and $8.40 \mathrm{kA} / \mathrm{m}(5.34 \%)^{a}$ for $\left\langle M_{x}\right\rangle$ and $\left\langle M_{y}\right\rangle$, respectively. Simulations are computed with $\mathrm{M}^{3} \mathrm{~S}$.

${ }^{a}$ Percentage values are related to the maximum values of $\left|\left\langle M_{x}\right\rangle\right|=$ $281.61 \mathrm{kA} / \mathrm{m}$ and $\left|\left\langle M_{y}\right\rangle\right|=157.43 \mathrm{kA} / \mathrm{m}$

the $y$-component of the spatially averaged magnetization $\left\langle M_{y}\right\rangle$. This enables one to distinguish between errors in the adiabatic and the non-adiabatic term. These linear changes are also in agreement with Eq. (B1). 


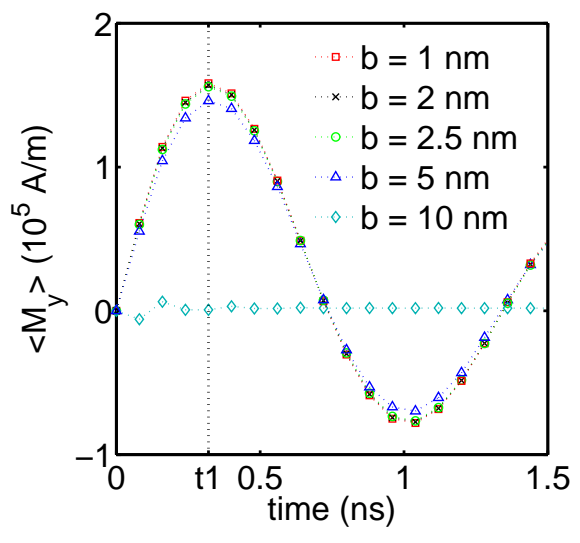

(a)



(b)

FIG. 4: (a) Spatially averaged magnetization component $\left\langle M_{y}\right\rangle$ for different cell sizes $b^{3}$ computed with $\mathrm{M}^{3} \mathrm{~S}$. (b) The $y$-component of the spatially averaged magnetization component $\left\langle M_{y}\right\rangle$ at time $t_{1}=0.32$ ns versus $b$.

\section{Improper Spatial Discretization}

To investigate the influence of the spatial discretization, we vary the number of discretization points of the FDM and FEM meshes. A FDM mesh is a grid that consists of equally sized cuboids (so-called discretization cells). FEM meshes, in contrast, cannot be described that simply, because here the size of each finite element can vary. To investigate the influence of the spatial discretization, we simulated the problem for five different cell sizes using the FDM-based tool $\mathrm{M}^{3} \mathrm{~S}$. The cell sizes used were $b \times b \times b$, for $b=1,2,2.5,5$, and $10 \mathrm{~nm}$. Figure 4(a) shows the time evolution of the $y$-component of the spatially averaged magnetization for the different cell sizes. Results for cell sizes $b=1,2,2.5$, and $5 \mathrm{~nm}$ show a slight decrease of the spatially averaged magnetization with increasing cell size. For a cell size of $b=10 \mathrm{~nm}$, no vortex is formed, i.e., criterion (iii) is not fulfilled. Fig- ure 4(b) shows the $y$-component of the spatially averaged magnetization at time $t=0.32 \mathrm{~ns}$ versus cell size $b$ fitted by a quadratic function. The extrapolation to $b=0$ suggests that it is sufficient to take a FDM mesh with a cell size of $2 \times 2 \times 2 \mathrm{~nm}^{3}$.

We have also simulated the problem for four FEM meshes using the FEM-based tool Nmag. Readers interested in FEM meshing can find a detailed description of the meshes used in the FEM simulations in appendix C. In the following, we use the maximum rod length and the number of tetrahedra as characteristic measures for the fineness of a mesh. The simulations with Nmag are performed with maximum rod lengths of 1.77, 2.36, 4.40, and $6.40 \mathrm{~nm}$, corresponding to 355488, 150282, 25560, and 8874 tetrahedra, respectively. Figure 5(a) shows the time evolution of the $y$-component of the spatially averaged magnetization for the different meshes. The results reveal a slight decrease of the precession frequency with increased rod length. Figure 5(b) shows the duration of the first gyration cycle for the rod length extrapolated to $0 \mathrm{~nm}$ by a quadratic function. The extrapolation suggests that it suffices to take a FEM mesh with a rod length of $2.36 \mathrm{~nm}$.

In accordance with the simulations of standard problem numbers $1-4^{26}$ these results illustrate, that to obtain reliable numerical results the distance between two discretization points should be significantly below the exchange length $l_{\mathrm{ex}}$.

\section{COMPARISON OF EXISTING TOOLS}

We compare the simulation results of OOMMF extended by Krüger et al. ${ }^{27}$, of OOMMF extended by Vanhaverbeke et al. ${ }^{32,33}$, of $\mathrm{M}^{3} \mathrm{~S}^{20}$, and of $\mathrm{Nmag}^{21}$. The results of both OOMMF-extensions and of $\mathrm{M}^{3} \mathrm{~S}$ have been computed using a cell size of $2 \times 2 \times 2 \mathrm{~nm}^{3}$, whereas the results of Nmag are computed using a mesh of type (i) as described in appendix $\mathrm{C}$ with a maximum rod length of $1.77 \mathrm{~nm}$. The corresponding regular mesh has 68211 mesh nodes, of which 17566 are surface nodes. The time evolution of the magnetization is performed by explicit or implicit numerical integration algorithms. Both tools, the spin-transfer torque extended OOMMF version of Krüger et al. ${ }^{27}$ and $\mathrm{M}^{3} \mathrm{~S}^{20}$, use an implementation of a fifth-order Cash-Karp Runge-Kutta algorithm ${ }^{34}$ with an absolute error tolerance of $10^{-3} \mathrm{~A} / \mathrm{m}$ and a relative error tolerance of $10^{-4}$. The spin-transfer torque extended OOMMF version of Vanhaverbeke et al. ${ }^{32,33}$ uses a fifth-order Dormand-Prince Runge-Kutta algorithm ${ }^{35}$ with the same error tolerances. Nmag uses the Sundials libraries ${ }^{36}$ with an absolute error tolerance of $8 \cdot 10^{-2}$ $\mathrm{A} / \mathrm{m}$ and a relative error tolerance of $10^{-7}$. Figure 6 shows the time evolution of the magnetization for all tools, whereas in table I the spatially averaged magnetization components for the relaxed state are listed. For comparison we also plot the analytically calculated values according to Krüger et al. ${ }^{27}$, which is explained in more 


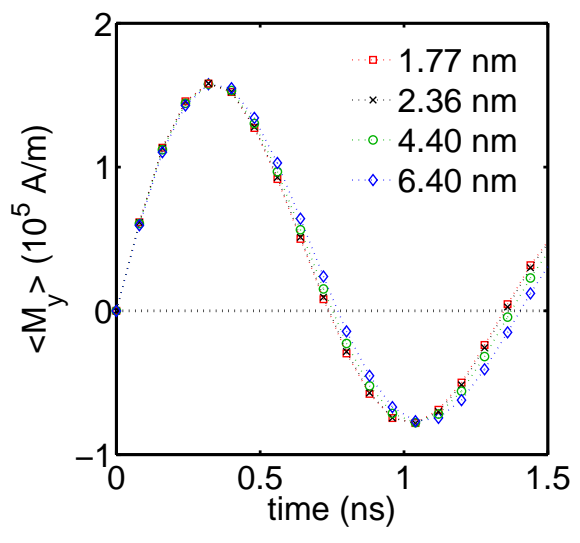

(a)



(b)

FIG. 5: Results for different FEM meshes computed with $\mathrm{Nmag}^{21}$. As maximum rod lengths $1.77,2.36,4.40$ and 6.40 $\mathrm{nm}$ are chosen which corresponds to $355488,150282,25560$, and 8874 tetrahedra, respectively. (a) Spatially averaged magnetization $\left\langle M_{y}\right\rangle$, (b) Duration of the first gyration cycle versus rod length.

detail in appendix B. The maximum difference of the spatially averaged magnetization between the simulation tools amounts to $5.41 \mathrm{kA} / \mathrm{m}(1.9 \%)^{47}(3.0 \%)^{47}$ for $\left\langle M_{x}\right\rangle$ and $\left\langle M_{y}\right\rangle$, respectively. In comparison to the analytical model, these differences are $16.14 \mathrm{kA} / \mathrm{m}(5.7 \%)^{47}$ and $11.27 \mathrm{kA} / \mathrm{m}(7.2 \%)^{47}$ for $\left\langle M_{x}\right\rangle$ and $\left\langle M_{y}\right\rangle$, respectively. We believe that the differences between the results in Fig. 6 are due to the implementation of the demagnetization field. A comparison of the simulation results of OOMMF and $\mathrm{M}^{3} \mathrm{~S}$ for standard problem number $4^{26}$ shows that they only differ in the calculation of the demagnetization field ${ }^{37}$. The spatially averaged magnetization of both OOMMF extensions are virtually identical but differ more significantly from $\mathrm{M}^{3} \mathrm{~S}$. Both $\mathrm{M}^{3} \mathrm{~S}$ and the OOMMF extensions use a demagnetization field implementation based on Newel et al. ${ }^{38}$. Unlike $\mathrm{M}^{3} \mathrm{~S}, \mathrm{OOMMF}$

\begin{tabular}{lcc}
\hline \hline Tools & $\begin{array}{c}\left\langle M_{x}\right\rangle \\
\left(10^{5} \mathrm{~A} / \mathrm{m}\right)\end{array}$ & $\begin{array}{c}\left\langle M_{y}\right\rangle \\
\left(10^{4} \mathrm{~A} / \mathrm{m}\right)\end{array}$ \\
\hline OOMMF+STT - Krüger & -1.71 & 1.51 \\
OOMMF+STT - Vanhaverbeke & -1.71 & 1.50 \\
$\mathrm{M}^{3} \mathrm{~S}$ - Najafi & -1.71 & 1.50 \\
NMag - Fangohr & -1.72 & 1.52 \\
Analytical model - Krüger & -1.78 & 1.12 \\
\hline \hline
\end{tabular}

TABLE I: Spatially averaged magnetizations $\left\langle M_{x}\right\rangle$ and $\left\langle M_{y}\right\rangle$ for the simulation tools and the analytical model at $t=14$ ns when the vortex has reached the new equilibrium position. All values in the table are rounded to two decimal places.

in addition uses an interpolation method to speed up the calculation of the demagnetization tensor. The FEMbased spatial discretization computes the demagnetization field with the hybrid finite element / boundary element method described by Fredkin and Koehler ${ }^{39}$. The difference between the numerical and the analytical results are a direct consequence of the approximations of the underlying analytical model, as explained in appendix B.

These results verify the suitability of the proposed standard problem, as the problem discriminates errors larger than about $3 \%^{47}$ and, in contrast to standard problem number 4 , no point of discontinuity is identified.

\section{EXPERIMENTAL FEASIBILITY}

Although not required for the proof of the micromagnetic simulations, it is nevertheless important to choose a problem that can be proved by experiments. Permalloy cuboids that exhibit the simulated magnetization configuration shown in Fig. 1 and 2 including wires contacting their left and right edges can be fabricated by electronbeam lithography and lift-off processing ${ }^{15}$. Experimentally it is a challenge to apply current densities in the $10^{12} \mathrm{~A} / \mathrm{m}^{2}$ regime permanently because of the concomittant large Joule heating. However, recently this problem has been solved by the preparation of permalloy nanostructures on diamond substrates ${ }^{40}$ : The diamond serves as a highly efficient heat sink, and it has been demonstrated that current densities in excess of $10^{12} \mathrm{~A} / \mathrm{m}^{2}$ can be applied continuously to samples like the one required for the proposed standard problem. The detection of the vortex core at the shifted position could, for example, be performed by scanning-electron microscopy with polarization analysis (SEMPA) ${ }^{41,42}$. As SEMPA detects the final steady-state position of the vortex core, the value of the damping constant $\alpha=0.1$ used in the simulation is not relevant. The degree of non-adiabaticity $\xi=0.05$ is a realistic experimental value ${ }^{43}$. As so far no experimental results of the proposed sample geometry are available, we validate the results of the micromagnetic simulations with the analytical model explained in detail in appendix 


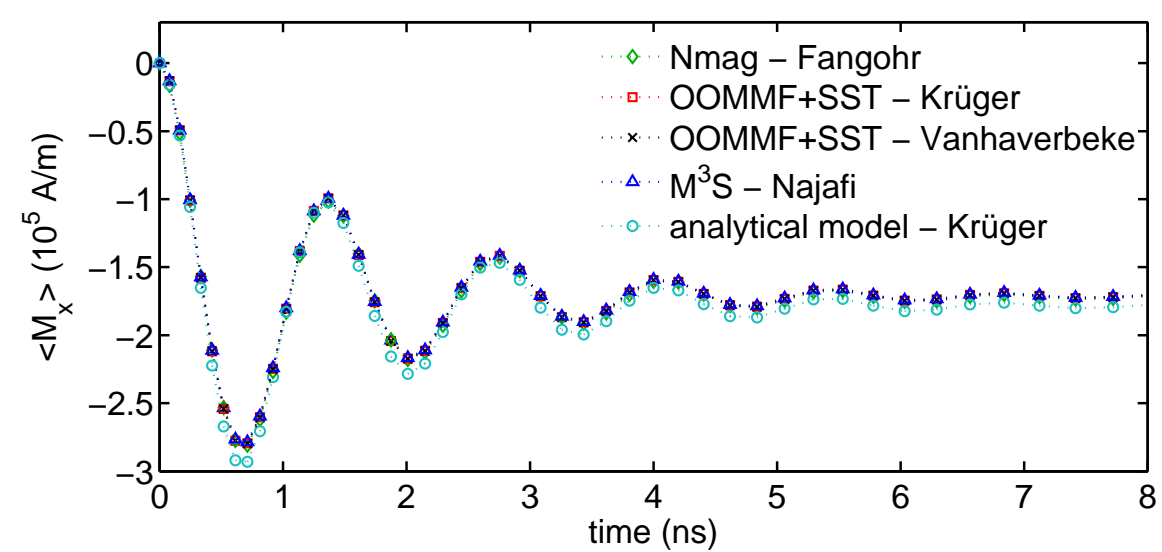

(a)

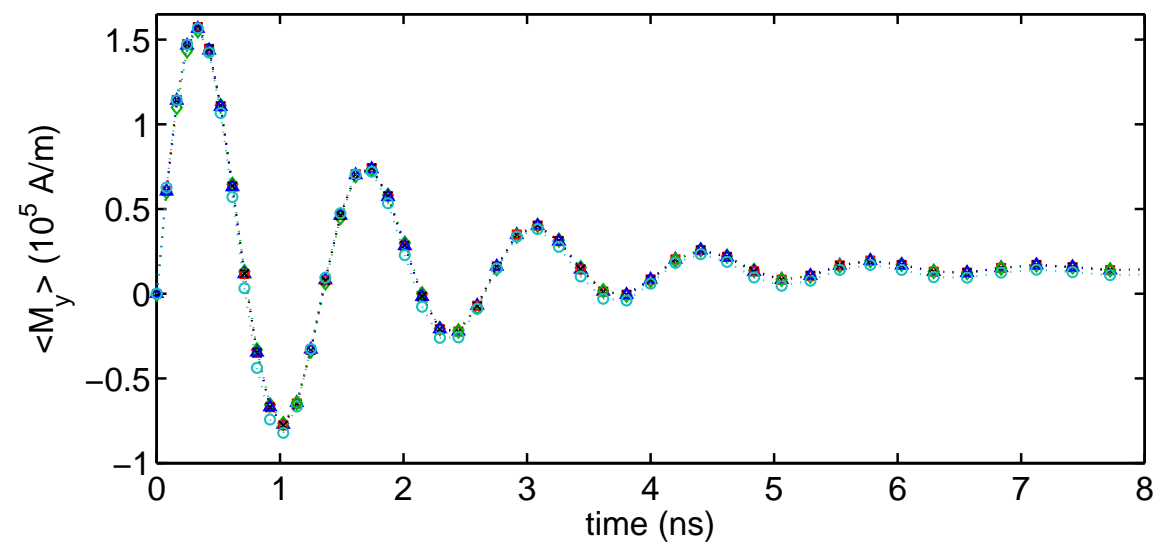

(b)

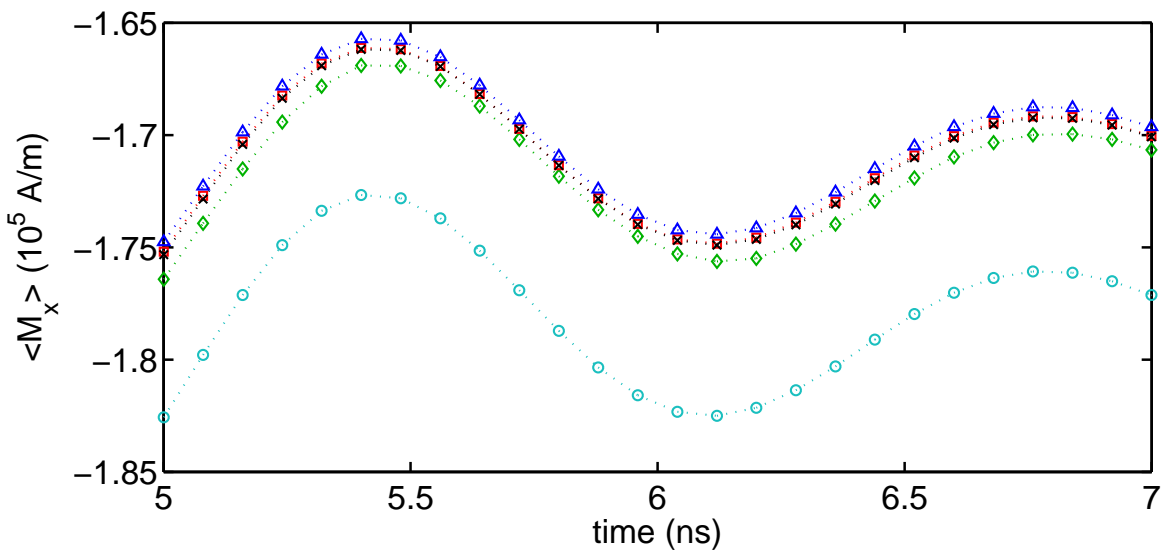

(c)

FIG. 6: Solution of the proposed standard problem for a $100 \times 100 \times 10 \mathrm{~nm}^{3}$ permalloy cuboid calculated with four different simulation tools and the analytical model. A spatially and temporally homogeneous current density of $10^{12} \mathrm{~A} / \mathrm{m}^{2}$ is applied instantaneously in $x$-direction. (a) The $x$-component of spatially averaged magnetization $\left\langle M_{x}\right\rangle$ (b) $\left\langle M_{y}\right\rangle$. (c) Close-up of the $x$-component $\left\langle M_{x}\right\rangle$ for the time interval $5 \mathrm{~ns} \leq t \leq 7 \mathrm{~ns}$. 
B. This model can serve as a reference because it has been already verified by experimental results on similar device geometries ${ }^{15}$.

\section{CONCLUSION}

In this work we present a standard problem for micromagnetic simulation packages extended by the spintransfer torque. For this standard problem, we defined the criteria necessary to ensure that the problem is suitable for the validation and falsification of micromagnetic simulation tools. These criteria have been applied to the underlying extended micromagnetic model. We have demonstrated that the standard problem has the required properties. To prove the good validation and falsification properties, we investigated the influence of typical errors, such as erroneous variations of the spin-transfer torque extension by a constant factor or an improper spatial discretization. The final comparison of the results for different tools substantiates these properties and shows that the problem discriminates errors larger than $5.41 \mathrm{kA} / \mathrm{m}$ $(1.9 \%)^{47}$ and $4.80 \mathrm{kA} / \mathrm{m}(3.0 \%)^{47}$ for $\left\langle M_{x}\right\rangle$ and $\left\langle M_{y}\right\rangle$, respectively.

\section{Acknowledgments}

Financial support by the Deutsche Forschungsgemeinschaft via the Graduiertenkolleg 1286 "Functional metalsemiconductor hybrid systems" and via the SFB 668 "Magnetism from single atoms to nanostructures", by the EPSRC (EP/E040063/1, EP/E039944/1), and by the ESF EUROCORES collaborative research project SpinCurrent under the Fundamentals of Nanoelectronics programme is gratefully acknowledged.

\section{APPENDIX A: RELATION BETWEEN SPATIALLY AVERAGED MAGNETIZATION AND VORTEX-CORE POSITION}

To show the correspondence of the vortex-core position and the spatially averaged magnetization, we use the model introduced by Krüger et al. ${ }^{27}$, where the vortex is described by four triangles $t_{1}$ to $t_{4}$ shown in Fig. 7 . The magnetization in each triangle is assumed to be homogeneous. If the vortex core is in the center of the cuboid, all four triangles have the same volume. As $t_{1}$ and $t_{3}$ as well as $t_{2}$ and $t_{4}$ have an antiparallel magnetization, the spatially averaged magnetization is zero. A deflection of the vortex core from the center of the cuboid changes the size of the triangles as illustrated in Fig. 7(b). The dependence of the spatially averaged magnetization on the volume differences and the deflection of the vortex core

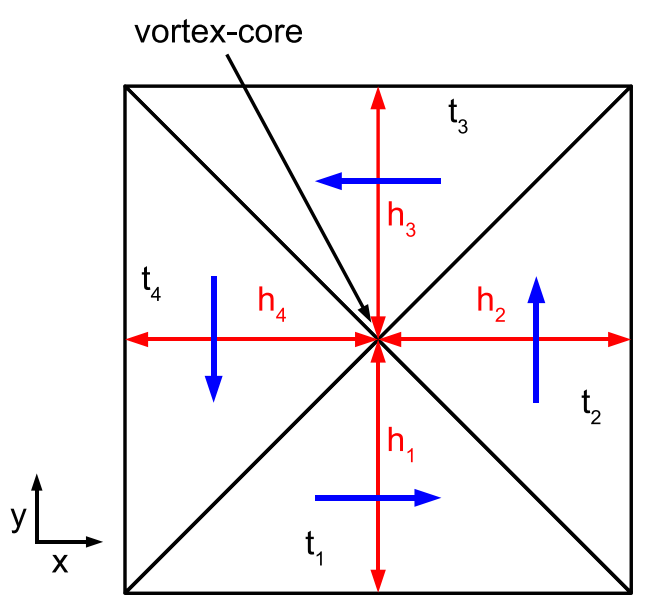

(a)

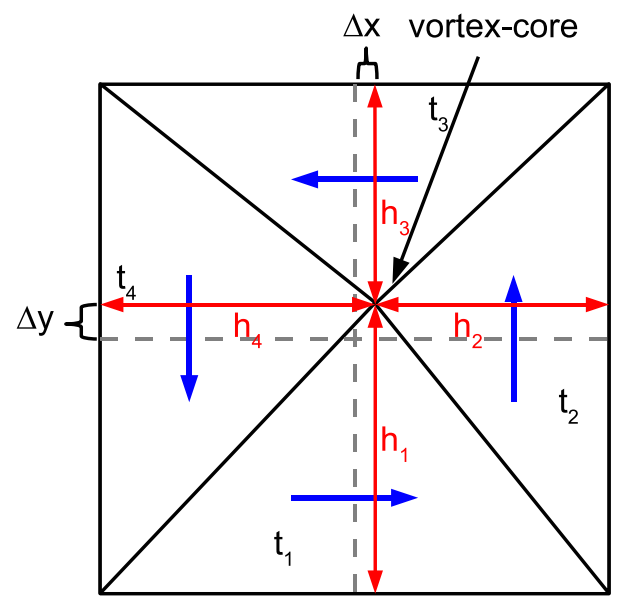

(b)

FIG. 7: Model for the vortex motion as introduced by Krüger et al. ${ }^{27}$. The magnetization pattern is described by four triangles $t_{1}$ to $t_{4}$. The vortex core is at the center of the four triangles. (a) Magnetization pattern with the vortex core at the center of the sample. (b) Magnetization configuration with a vortex core displaced from the center by $\Delta x$ and $\Delta y$.

is given by

$$
\begin{aligned}
\left(\begin{array}{c}
\left\langle M_{\mathrm{x}}\right\rangle \\
\left\langle M_{\mathrm{y}}\right\rangle \\
\left\langle M_{\mathrm{z}}\right\rangle
\end{array}\right) & =\left(\begin{array}{c}
c M_{\mathrm{s}} k \cdot \frac{V_{1}-V_{3}}{V_{\text {cuboid }}} \\
c M_{\mathrm{s}} k \cdot \frac{V_{2}-V_{4}}{V_{\text {cuboid }}} \\
p \cdot \text { const }
\end{array}\right)=\left(\begin{array}{c}
c M_{\mathrm{s}} k \cdot \frac{l d \Delta y}{l^{2} d} \\
c M_{\mathrm{s}} k \cdot \frac{l d\left(\frac{\Delta x)}{l^{2} d}\right.}{p \cdot \operatorname{const}}
\end{array}\right) \\
& =\left(\begin{array}{c}
c M_{\mathrm{s}} k \cdot \frac{\Delta y}{l} \\
-c M_{\mathrm{s}} k \cdot \frac{\Delta x}{l} \\
p \cdot \text { const }
\end{array}\right) .
\end{aligned}
$$

Here $V_{\mathrm{i}}$ is the volume of triangle $t_{i}, l$ the edge length of the cuboid, $d$ its thickness, $c$ the chirality of the magnetization pattern, $p$ the polarization of the vortex, $\Delta x=\left(h_{4}-h_{2}\right) / 2$ the deflection of the vortex core in $x$ direction, $\Delta y=\left(h_{1}-h_{3}\right) / 2$ the deflection in $y$-direction, and $h_{i}$ the height of triangle $t_{i}$. The dimensionless fit 
parameter $k$ is needed to convert the vortex-core position into the spatially averaged magnetization, and takes into account that the domain walls between the triangles in Fig. 7 have a finite size and are not abrupt as treated in Eq. (A1). The value of $k$ changes with the system size and is 1.4517 for the proposed geometry. Because of the cuboid geometry, the $x$-component of the spatially averaged magnetization $\left\langle M_{x}\right\rangle$ is proportional to the deflection $\Delta y$ of the vortex core in the $y$-direction and the $y$-component of the spatially averaged magnetization $\left\langle M_{y}\right\rangle$ is proportional to the deflection $\Delta x$ in the $x$-direction.

\section{APPENDIX B: ANALYTICAL MODEL}

The vortex-core position can be calculated by the analytical model described $\mathrm{in}^{27}$. This model is in accordance with experimental results on the spin-transfer torque ${ }^{15}$. For a square, the model predicts that the final deflection of the vortex core in the $x$-direction depends only on the non-adiabatic spin-transfer torque term and that the final deflection in $y$-direction depends only on the adiabatic spin-transfer torque term:

$$
\left(\begin{array}{c}
\Delta x_{\text {end }} \\
\Delta y_{\text {end }}
\end{array}\right)=-\left(\begin{array}{c}
\frac{b_{j} j \Gamma \xi}{\alpha\left(\omega^{2}+\Gamma^{2}\right)} \\
\frac{b_{j} j \omega}{\omega^{2}+\Gamma^{2}}
\end{array}\right)
$$

Here $\omega$ is the free frequency of the gyration of the vortex core, $\Gamma$ is the damping constant of the vortex, $\alpha$ is the Gilbert damping constant, and $\left(\Delta x_{\text {end }}, \Delta y_{\text {end }}\right)$ is the final position of the vortex core related to the center of the cuboid. The time evolution of the core's position

$$
\begin{aligned}
& \left(\begin{array}{c}
\Delta x(t) \\
\Delta y(t)
\end{array}\right)= \\
& \left(\begin{array}{c}
A i e^{(-\Gamma+i \omega) t}-B i e^{(-\Gamma-i \omega) t}+\Delta x_{\text {end }} \\
A e^{(-\Gamma+i \omega) t}+B e^{(-\Gamma-i \omega) t}+\Delta y_{\text {end }}
\end{array}\right)
\end{aligned}
$$

depends on the coefficients $A=\left(-\Delta y_{\text {end }}+i \Delta x_{\text {end }}\right) / 2$ and $B=\left(-\Delta y_{\text {end }}-i \Delta x_{\text {end }}\right) / 2$. Owing to approximations within the analytical model concerning the detailed magnetization pattern a perfect agreement with the micromagnetic simulations cannot be expected.

\section{APPENDIX C: USED FINITE ELEMENT MESHES}

We have used two different types of finite-element meshes in the calculations with $\mathrm{Nmag}^{21}$ :

1. meshes created by decomposing the cuboidal body into cubes,

2. meshes generated with the advancing front method using NETGEN ${ }^{44}$.

For method (i), each cube is subdivided into six tetrahedra consistently with the neighboring cubes. The cubes are then skewed to obtain nearly equilateral triangles on the surface of the mesh. We keep only those tetrahedra that lie within the ferromagnetic region and adjust those that intersect the meshing region surface (the points outside the meshing region are projected back onto its surface). The advantages of using this "regular mesh" are that all edge lengths are exactly known and that the mesh generation is very fast for the cuboidal geometry. For the unstructured tetrahedral mesh (ii), we use the mesh generator NETGEN ${ }^{44}$, which is based on the advancing front method. The results of Nmag in Section IV have been computed using a mesh of type (i) with a maximum edge length of $1.77 \mathrm{~nm}$ that has 68211 mesh nodes, of which 17566 are surface nodes. This has been compared with an unstructured mesh generated with Netgen with 25887 points and rod lengths varying from $1 \mathrm{~nm}$ to $3.8 \mathrm{~nm}$, with an average rod length of $1.95 \mathrm{~nm}$. The simulation results are virtually independent of the mesh types used.
* Electronic address: mnajafi@physnet.uni-hamburg.de

$\dagger$ Electronic address: bkrueger@physnet.uni-hamburg.de

1 D. A. Thompson and J. S. Best, IBM J. Res. Develop. 44, 311 (2000).

2 International technology roadmap for semiconductors 2007 edition, Semiconductor Industry Association (2007), http://www.itrs.net/Links/2007ITRS/Home2007.htm.

3 T. M. Maffitt, J. K. DeBrosse, J. A. Gabric, E. T. Gow, M. C. Lamorey, J. S. Parenteau, D. R. Willmott, M. A. Wood, and W. J. Gallagher, IBM J. Res. Develop. 50, 25 (2006).

4 M. Jullière, Phys. Lett. A 54, 225 (1975).

${ }^{5}$ G. Binasch, P. Grünberg, F. Saurenbach, and W. Zinn, Phys. Rev. B 39, 4828 (1989).
${ }^{6}$ E. C. Stoner and E. P. Wohlfarth, Phil. Trans. Royal Soc. London A240, 599 (1948).

7 C. Chappert, A. Fert, and F. N. Van Dau, Nature Materials 6, 813 (2007).

8 J. Slonczewski, J. Mag. Mag. Mat. 159, 1 (1996).

9 J. Slonczewski, J. Mag. Mag. Mat. 247, 324 (2002).

10 L. Berger, Phys. Rev. B 54, 9353 (1996).

11 M. Hosomi, H. Yamagishi, T. Yamamoto, K. Bessho, Y. Higo, K. Yamane, H. Yamada, M. Shoji, H. Hachino, C. Fukumoto, et al., Electron Devices Meeting, 2005. IEDM Technical Digest. IEEE International p. 459 (2005).

12 S. S. P. Parkin, M. Hayashi, and L. Thomas, Science 320, 190 (2008).

13 S. Zhang and Z. Li, Phys. Rev. Lett. 93, 127204 (2004). 
14 A. Thiaville, Y. Nakatani, J. Miltat, and Y. Suzuki, Europhys. Lett. 69, 990 (2005).

15 M. Bolte, G. Meier, B. Krüger, A. Drews, R. Eiselt, L. Bocklage, S. Bohlens, T. Tyliszczak, A. Vansteenkiste, B. Van Waeyenberge, et al., Phys. Rev. Lett. 100, 176601 (2008).

16 S. Bohlens, B. Krüger, A. Drews, M. Bolte, G. Meier, and D. Pfannkuche, Appl. Phys. Lett. 93, 142508 (2008).

17 J. A. Mandelman, R. H. Dennard, G. B. Bronner, J. K. DeBrosse, R. Divakaruni, Y. Li, and C. J. Radens, IBM J. Res. Develop. 46, 187 (2002).

18 R. W. Mann, W. W. Abadeer, M. J. Breitwisch, O. Bula, J. S. Brown, B. C. Colwill, P. E. Cottrell, W. G. Crocco, S. S. Furkay, M. J. Hauser, et al., IBM J. Res. Develop. 47, 553 (2003).

19 A. Vladimirescu, The SPICE book (Wiley, New York, 1994).

${ }^{20}$ M. Najafi, B. Krüger, S. Bohlens, G. Selke, B. Güde, M. Bolte, and D. P. F. Möller, GCMS 08: Proceedings of the 2008 Conference on Grand Challenges in Modeling \& Simulation p. 427 (2008).

21 T. Fischbacher, M. Franchin, G. Bordignon, and H. Fangohr, IEEE Trans. Magn. 43, 2896 (2007).

22 M. J. Donahue and D. G. Porter, National Institute of Standards and Technology, Gaithersburg, MD 6376 (1999).

23 M. R. Scheinfein, LLG-micromagnetics simulator (2008), http://llgmicro.home.mindspring.com/.

${ }^{24}$ D. V. Berkov, MicroMagus - software for micromagnetic simulation (2008), http://www.micromagus.de.

25 W. F. Brown Jr., Micromagnetics (Interscience Publishers, New York, 1963).

${ }^{26}$ Micromagnetic Modeling Activity Group - $\mu M a g, \quad$ National Institute of Standards and Technology, Gaithersburg, MD (2008), http://www.ctcms.nist.gov/ rdm/mumag.org.html.

27 B. Krüger, A. Drews, M. Bolte, U. Merkt, D. Pfannkuche, and G. Meier, Phys. Rev. B 76, 224426 (2007).

28 B. Krüger, D. Pfannkuche, M. Bolte, G. Meier, and U. Merkt, Phys. Rev. B 75, 054421 (2007).

29 S.-K. Kim, K.-S. Lee, Y.-S. Yu, and Y.-S. Choi, Appl. Phys. Lett. 92, 022509 (2008).

30 K.-S. Lee, Y.-S. Yu, Y.-S. Choi, D.-E. Jeong, and S.-K.
Kima, Appl. Phys. Lett. 92, 192513 (2008).

31 A. Hubert and R. Schäfer, Magnetic Domains: The Analysis of Magnetic Microstructures (Springer, Berlin, 1998).

32 A. Vanhaverbeke, OOMMF extension of spin-transfer torque terms for currentinduced domain wall motion (2008), http://www.zurich.ibm.com/st/magnetism/spintevolve.html.

33 A. Vanhaverbeke, A. Bischof, and R. Allenspach, Phys. Rev. Lett. 101, 107202 (2008).

34 J. R. Cash and A. H. Karp, ACM Trans. Math. Softw. 16, 201 (1990).

35 J. R. Dormand and P. J. Prince, J. Comput. Appl. Math. 6, 19 (1980).

36 Sundials libraries (2008), http://acts.nersc.gov/sundials/index.html.

37 M.-A. B. W. Bolte, M. Najafi, G. Meier, and D. P. F. Möller, SCSC 07:Proceedings of the 2007 Summer Computer Simulation Conference (2007).

38 A. Newell, W. Williams, and D. Dunlop, J. Geophys. Res. 98 p. 9551 (1993).

39 D. R. Fredkin and T. R. Köhler, IEEE Trans. Magn. 26, 415 (2000).

40 S. Hankemeier, K. Sachse, Y. Stark, R. Frömter, and H. P. Oepen, Appl. Phys. Lett. 92, 242503 (2008).

41 R. Allenspach and P.-O. Jubert, MRS Bull. 31, 395 (2006).

${ }^{42}$ H. Hopster and H. P. Oepen, Magnetic Microscopy of Nanostructures (Springer, Berlin, 2004).

43 S. Lepadatu, M. C. Hickey, A. Potenza, H. Marchetto, T. R. Charlton, S. Langridge, S. S. Dhesi, and C. H. Marrows (2008).

44 J. Schoberl, Computing and Visualization in Science 1, 41 (1997).

45 Intel microprocessors http://www.intel.com/support/processors/sb/CS023143.htm.

46 The computation time is measured with a simulation run on a computer containing a Intel-Core-Duo-E6600 microprocessor with a performance of 19.20 GFlops $^{45}$. The simulation uses one core.

47 Percentage values are related to the maximum values of $\left|\left\langle M_{x}\right\rangle\right|=281.61 \mathrm{kA} / \mathrm{m}$ and $\left|\left\langle M_{y}\right\rangle\right|=157.43 \mathrm{kA} / \mathrm{m}$ and $4.80 \mathrm{kA} / \mathrm{m}$. 